\title{
Genipin Diffusion and Reaction into a Gelatin Matrix for Tissue Engineering Applications
}

Francesca Montemurro a , Carmelo De Maria a ${ }^{\text {a, }}$, Gianni Orsi a ${ }^{\text {a }}$ Lisa Ghezzi ${ }^{\text {b }}$, Maria Rosaria Tinè ${ }^{\text {, }}$ Giovanni Vozzi ${ }^{\text {a,c }}$

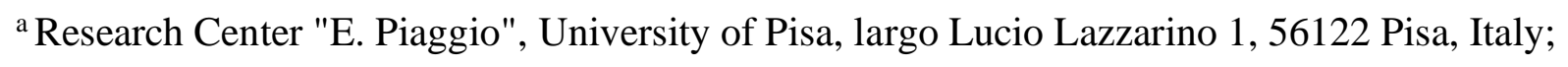

${ }^{\mathrm{b}}$ Department of Chemistry and Industrial Chemistry, University of Pisa, via G. Moruzzi 3, 56124 Italy.

${ }^{c}$ Department of Information Engineering, University of Pisa, via G. Caruso 16, 56122 Italy.

*corresponding author:

E-Mail: carmelo.demaria@ @entropiaggio.unipi.it;

Tel.: +39-050-2217056;

Fax: +39-050-2217051. 


\section{Abstract}

Genipin is a natural low-toxic crosslinker for molecules with primary amino groups, and its use with collagen and gelatin has shown a great potential in tissue engineering applications. The fabrication of scaffolds with a well-organized micro and macro topology using additive manufacturing systems requires an accurate control of working parameters, such as reaction rate, gelling time and diffusion constant. A polymeric system of $5 \%$ w/v gelatin in PBS with $2 \mathrm{mg} / \mathrm{ml}$ collagen solutions in a 1:1 weight ratio was used as template to perform measurements varying genipin concentration in a range $0.1-1.5 \% \mathrm{w} / \mathrm{w}$ respect to gelatin. In the first part of this work, the reaction rate of the polymeric system was estimated using a new colorimetric analysis of the reaction. Then its workability time, closely related to the gelling time, was evaluated thanks to rheological analysis: Finally, the quantification of static and dynamic diffusion constants of genipin across non-reacting and reacting membranes, made respectively by agarose and gelatin, was performed.

It was shown that the colorimetric analysis is a good indicator of the reaction progress. The gelling time depends on the genipin concentration, but a workability window of 40 minutes guaranteed up to $0.5 \% \mathrm{w} / \mathrm{w}$ genipin. The dynamic diffusion constant of genipin in the proposed polymeric system is in the order of magnitude of $10^{-7}$. The obtained results indicated the possibility to use the genipin, gelatin and collagen, in the proposed concentrations, to build well-defined hydrogel scaffolds both with extrusion-based and 3D ink-jet system.

\section{Keywords}

Genipin; gelatin; collagen; reaction rate; viscosity; diffusion constant; colorimetric evaluation; Biofabrication. 


\section{Introduction}

Tissue Engineering (TE) strategies have potential to be used in the regeneration of a series of soft tissues and organs, such as articular cartilage, skeletal muscle, cardiac and hepatic tissue. Natural polymers are the most suitable to promote cell function, such as adhesion, proliferation and differentiation, because they contain biochemical cues able to mimic extracellular matrix (ECM); unfortunately, at the same time, they could cause antigenicity [1-3].

Collagen-based materials are of special interest, because collagen is the major insoluble fibrous protein of ECM. In addition to its structural, collagen promotes cell answer to soluble factors during tissue remodelling and repair, and participates to wound healing processes and platelet aggregation $[1,3-5]$.

Gelatin is obtained by thermal denaturation or physical and chemical degradation of collagen, which induce the breaking of its triple-helix structure. It is widely used in the pharmaceutical industry as well as in biomedical area: hard and soft capsules, microspheres, sealants for vascular prostheses, wound dressing and adsorbent pads for surgical use are some of its most frequent applications.

Because collagen-based materials are rapidly digested in vivo, their degradation in the human body must be controlled until the tissue regeneration process is finished. This result is usually achieved through cross-linking agents [4-6]. Commonly used chemical cross-linkers include formaldehyde [4,6], glutaraldehyde [4,7], polyepoxy compounds [7,8], carbodiimides [8,9] and acyl azide [9-11]. However, these synthetic cross-linking reagents are relatively high cytotoxic, impairing the biocompatibility of the crosslinked material [10-14].

Genipin (GP) is an alternative natural cross-linker that presents a low-toxic level [12-14]: it is 10000 times less cytotoxic than glutaraldehyde $[12,15]$, and it can form stable cross-linked products 
with resistance against enzymatic degradation that is comparable to that of glutaraldehyde-fixed tissue $[15,16]$. GP and its related iridoid glucosides are extracted from the fruits of Gardenia jasminoides Ellis. The fruit is an oriental folk medicine, which has been included in traditional formulations. The pharmacological actions of the whole fruit, such as protective activity against oxidative damage, cytotoxic effect, anti-inflammatory activity and fibrolytic activity have already been elucidated $[12,16,17]$.

GP reacts with materials containing primary amine groups, such as chitosan, gelatin and some peptides and polypeptides, to form covalently crosslinked networks $[12,17,18]$. The crosslinking process happens through a series of reactions involving different sites on GP molecule ending with a radical polymerization responsible of blue pigment of final product. Thanks to radical reactions GP is able to establish long-range intermolecular crosslinking respect to the other crosslinkers such as carbodiimides and N-hydroxysuccinimide (NHS), or glutaraldehyde [18-20], which present intramolecular and short-range intermolecular crosslinks. The reaction mechanism of amino-groupcontaining compounds with GP has been discussed in literature [19-20]. It has been proposed that a GP-amino-group monomer is formed through a nucleophilic attack by amino-group-containing compounds such as gelatin on the third carbon of GP. The opening of the dihydropyran ring is then followed by attack on resulting aldehyde group by secondary amine formed in the first step of reaction. An heterocyclic compound of GP linked to residues containing primary amine groups (present in the gelatin chain) is thereby formed. The polymerization reactions are induced by the presence of oxygen radicals. When the radical reaction starts, GP molecules react with one (a dimer) or more GP molecules before crosslinking with amino group-containing compounds.

As scaffold material, GP -crosslinked genipin was used in chondrogenic differentiation of human adipose mesenchimal stem cells [21,22], nerve repair [23], and for recreating the mechanical cues 
of satellite cells' niche in muscle tissue engineering [24]. Genipin, gelatin and collagen were used as organic matrix filled with hydroxyapatite for bone regeneration $[25,26]$.

Recently, the application of additive manufacturing technologies has given to TE researchers new degrees of freedom in scaffold fabrication. The biofabrication of hydrogel-based structures can be divided in methods based on laser-induced forward transfer (less common), inkjet printing and robotic dispensing. Although standardized tests to evaluate the capacity of hydrogels to be printed do not yet exist [27], some necessary features can be extrapolated.

In the robotic dispensing approach, hydrogels, usually inserted in disposable plastic syringes, are dispensed either pneumatic, piston- or screw-driven, on a building platform as strands. In order to retain the shape of the structure after printing, highly viscous hydrogels are often used. Furthermore the crosslinking time is another key parameter, in order to guarantee a reasonable trade-off between the fabrication time and the shape retention, especially in those cases in which the crosslinker is extruded together with the gelling material [28].

The 3D inkjet printing for hydrogel material is usually based on printing crosslinking droplets onto a gel substrate following a well-defined trajectory, in order to fabricate a topologically defined layer of crosslinked polymer. Repeating this approach layer-by-layer it is possible to build a 3D object with a custom geometry [29]. This fabrication method is mostly dependent on the crosslinker diffusion/reaction characteristics $[27,29,30]$, described by the dynamic diffusion constant.

The aim of the present work is to elucidate some properties of the gelling system formed by collagen-gelatin and GP in order to optimise the fabrication of hydrogel scaffolds for soft TE applications.

In the first part, the measure of the reaction rate of GP with gelatin and collagen, using a colorimetric analysis based on image processing method, was performed. Then the processing time 
was estimated on the basis of rheological analysis. Finally, the static and dynamic diffusion constants of the GP across a gel, using a purposely-designed experimental setup, were evaluated. 


\section{Materials and methods}

\section{Sample preparation to evaluate the reaction rate constant}

Gelatin type A from porcine skin was purchased from Sigma-Aldrich (Italy). The 5\% w/v gelatin solution was prepared dissolving gelatin in phosphate buffered saline (PBS) solution, heating and mixing it to $70^{\circ} \mathrm{C}$ on a stirrer for $1 \mathrm{~h}$ till the solute was totally dissolved. Collagen type I was extracted from rat tail $[31,32]$ with a $4 \mathrm{mg} / \mathrm{ml}$ concentration. GP (98\% by HPLC) was purchased from Challenge Bioproducts Co., Ltd (CBC, Taiwan). A series of samples were prepared mixing $5 \%$ w/v gelatin with different GP concentrations: $0.25 \%, 0.5 \%, 1.0 \%$ and $1.5 \% \mathrm{w} / \mathrm{v}$ of solution. Five samples for each GP concentration were prepared. The solutions were stirred to favour GP dissolution and let to crosslink in a 24 multiwell plate once at room temperature. Other samples were prepared mixing $5 \% \mathrm{w} / \mathrm{v}$ gelatin and $2 \mathrm{mg} / \mathrm{ml}$ collagen solutions in a 1:1 weight ratio (i.e. $1 \mathrm{~g}$ of gelatin with $1 \mathrm{~g}$ of collagen) and were crosslinked with the same GP concentrations as above described. Also in this case, five samples for each GP concentration were prepared.

\section{Image processing}

To evaluate the reaction rate constant, the formation of blue pigment was considered as an index of crosslinking progress. The reaction was followed for 48 hours and pictures were taken at fixed time. Pictures were imported as RGB matrices in Matlab ${ }^{\circledR}$ (The Mathworks Inc. USA) and processed. The mean value of the blue component of each matrix was extracted after image processing. The blue intensity values were plotted against time.

\section{Rheological analysis}

The rheological properties of the gelatin-collagen polymeric system were evaluated as function GP concentration by dynamic oscillatory testing using the Rheometer HAAKE Rheostress 6000 (ThermoScientific) equipped with cone - plate (cone angle $1^{\circ}$ ). Briefly, samples were prepared as indicated in the section above for the evaluation of the reaction rate constant: $5 \%$ w/v gelatin 
solution and $2 \mathrm{mg} / \mathrm{ml}$ collagen solution were mixed in a 1:1 weight ratio (i.e. $1 \mathrm{~g}$ of gelatin with $1 \mathrm{~g}$ of collagen) and crosslinked with the following GP concentrations: $0.1 \%, 0.25 \%, 0.5 \% \mathrm{w} / \mathrm{v}$. Three samples for each GP concentration were prepared. The temperature was set at $37^{\circ} \mathrm{C}$. The storage $\left(G^{\prime}\right)$ and loss $\left(G^{\prime \prime}\right)$ moduli were measured over time at a constant frequency $1 \mathrm{~Hz}$ and $10 \%$ strain. All samples were measured within the linear viscoelastic region. There are several methods to identify the gelling point [33]; in the present work the crossing point between G' and G', was used.

\section{Static and dynamic diffusion constant determination: diffusion chamber design}

With the aim at determining the static and dynamic diffusion constants, a custom made "diffusion device" was designed: it was composed of two adjacent chambers separated by thin removable Teflon septum (S) which held a thin membrane of agarose or gelatin, as shown in figure 1a.

Agarose for routine use with a gelling point between $34.5^{\circ} \mathrm{C}$ and $37.5^{\circ} \mathrm{C}$ was purchased from Sigma-Aldrich. A $1 \% \mathrm{w} / \mathrm{v}$ agarose solution was prepared in boiling deionized water and poured to solidify in the septum hole to form the membrane. The same experimental setting was followed for the gelatin membrane, prepared starting from a $5 \% \mathrm{w} / \mathrm{v}$ gelatin solution.

The larger chamber (LC) was $10 \mathrm{~cm} \times 10 \mathrm{~cm}$ and $1 \mathrm{~cm}$ in height, while the smaller one, hereafter named diffusion chamber (DC), was $5 \mathrm{~cm} \mathrm{x} 2 \mathrm{~cm}$ and $1 \mathrm{~cm}$ in height (figure 1a). The LC was filled with $100 \mathrm{ml}$ solution of $0.25 \%$ or $0.5 \%$ w/v GP in PBS, and the DC was filled with $10 \mathrm{ml}$ of PBS solution.

The septum ( $\mathrm{S}$ in figure 1a) was composed of three parts as shown in figure $1 \mathrm{~b}$. The first part (A in figure $1 \mathrm{~b}$ ), made in Teflon, had a central hole with a surface of $1 \mathrm{~cm}^{2}$. The second part (B in figure $1 \mathrm{~b}$ ) was a thin silicone film (1 mm thickness) which acted as support for the membrane. Finally the third part $(\mathrm{C}$ in figure $1 \mathrm{~b})$ was a Plexiglas skin, used as tightening to hold the part $\mathrm{B}$ 
pressed against the part A. All these parts had in the central area a window to allow diffusion. On A and $\mathrm{C}$, a large mesh gauze, to not interfere in the measurement, was glued to confine the membrane in the septum.

The design of the device was based on the following considerations: 1) the ratio 1:10 between solutions volume let the diffusion process reach the steady state quickly; 2) osmotic pressure is considered in inverse relation to volume, it had a low value because GP solution was initially in the LC. Samples were collected from the DC at fixed time at room temperature, and GP concentration was measured using FLUOstar Omega spectrofluorimeter (BMG Labtech, Ortenberg Germany) at wavelength of $244 \mathrm{~nm}$. Experiments were performed in triplicate.

\section{Statistical analysis}

Values were reported as mean \pm standard deviation. Statistical significance was assessed using the two tails t-test and the two way analysis of variance (two-way ANOVA), with a confidence level of $95 \%(\mathrm{p}<0.05)$. 


\section{Results}

\section{Reaction rate constant}

The GP crosslinking reaction with primary amine groups was established to be a second order reaction: the measured order was actually 1.78 , a value close to 2 , which was the expected value for a simple irreversible gelation process [34]. The present analysis started from the rate equation (eq. 1) of a general chemical equation $\mathrm{A}+\mathrm{B} \rightarrow \mathrm{P}$ (where $\mathrm{A}$ and $\mathrm{B}$ are the reagents and $\mathrm{P}$ is the product):

$$
v=\frac{d P}{d t}=k[A]^{m}[B]^{n}
$$

If we suppose that the reaction is of second order respect to A (which represents the GP) and zero respect to $\mathrm{B}$ (which represents the gelatin and the collagen), $\mathrm{P}$ represents the polymer formation after GP reaction; by the integration of the rate equation (eq.1) over the time we obtain (eq. 2):

$$
[P]=\frac{k t[A]_{0}^{2}}{1+k t[A]_{0}}
$$

where $[\mathrm{A}]_{0}$ is the initial concentration of A. After GP reaction, the polymeric system becomes blue and this change was considered an index of reaction progress; thus we expressed $[\mathrm{P}]$ as blue intensity. By rearranging the last equation, eq.3 was obtained:

$$
\frac{1}{[P]}=\frac{1}{k t\left[A_{0}\right]^{2}}+\frac{1}{\left[A_{0}\right]}
$$

By plotting $1 /[P]$ versus $1 / t$, a linear behaviour with slope $m=1 / k t\left[A_{0}\right]^{2}$ and with $y$-intercept $q=$ $1 /\left[A_{0}\right]$ was obtained (Figures. 2 and 3): for each sample the $\mathrm{k}$ value can by calculated from the slope and it is expressed as blue intensity ${ }^{-1} \min ^{-1}$ (Fig. 4). For convenience, raw data are listed in table 1. 
The two-way ANOVA statistical test (p-value $<0.05$ ) demonstrated that there are not significant differences both respect to the GP concentration and both respect to the presence of the collagen into the polymeric system.

\section{Rheological analysis}

In order to find the gelation time, the cross point between $G$ ' and G', was considered: as shown in figure 5 the gelling time decreases as the GP concentration increases.

In the biofabrication of well-defined structures using robotic dispensing technologies, the gel can be extruded also after this gelation point: very high viscosity can limit the workability and can bring to the gel rupture. Thus from practical point of view, it is very interesting to visualize the trend of viscosity over time (fig.6).

It is possible to note that there is no marked difference between the various polymeric systems before 40 minutes, and at low GP concentration $(0.1 \%$ and $0.25 \%)$ the trend is similar also until 60 minutes.

\section{Static and dynamic diffusion coefficient determination}

According to the structure of the diffusion device (fig. 1), $\mathrm{V}_{1}$ was the volume of LC and $\mathrm{C}_{1}$ the concentration of GP solution in this chamber, while $\mathrm{V}_{2}$ was the volume of the $\mathrm{DC}$ and $\mathrm{C}_{2}$ the concentration of the respective GP solution; L and A were the membrane thickness and its area respectively; D was the diffusion coefficient.

The system followed the Fick's laws, and at the steady state the flux was considered constant within membrane, due to its small thickness. From the mathematical analysis, provided in the additional information documentation, the concentration in the DC was (eq. 4):

$$
C_{2}=\frac{V_{1} C_{1}}{V_{1}+V_{2}} \alpha \cdot\left(1-e^{\frac{D \cdot A}{L} \cdot \frac{V_{1}+V_{2}}{V_{1} V_{2}} t}\right)
$$


To take into account the absorption of GP molecules on gelatin and agarose membrane, and to maintain an easier theoretical formulation, the coefficient $\alpha$ was introduced, that modified the steady state concentration. This phenomenon limited the molecules motility through the membrane, and the diffusion coefficient decreased with the time.

GP concentrations in diffusion chamber for agarose and gelatin membrane, measured by a spectrofluorimeter, were plotted versus time (fig. 7a-b and fig. 8a-b) and fitted with equation 4 to obtain the D (and $\alpha$ ), with a routine purposely implemented in Matlab ${ }^{\circledR}$ (The Mathworks Inc.) using the Weighed Least Square algorithm.

The static diffusion coefficient, obtained from experiments with the agarose membrane, is (3.69 $\pm 0.47) \cdot 10^{-6} \mathrm{~m}^{2} / \mathrm{s}$ with practically no statistically significant differences between the two concentrations of GP (T-test with a p-value <0.05).

The dynamic diffusion coefficient, obtained using the gelatin membrane, involved the crosslinking reaction between amino groups of gelatin and GP, is not statistically different (T-test) respect the two tested GP concentration, and it is as average $(9.06 \pm 0.31) \cdot 10^{-7} \mathrm{~m}^{2} / \mathrm{s}$.

The dynamic and static diffusion constants are statistically different, as demonstrated by performing the two-way ANOVA (p-value <0.05) on all the measurements on agarose and gelatin. 


\section{Discussion}

The GP crosslinking reaction with gelatin and collagen was characterized in terms of reaction rate constant, rheology and diffusion coefficients, in order to determine the working window of the most common additive manufacturing technologies for fabricating hydrogel scaffolds for TE applications.

The reaction rate was evaluated for several GP concentrations considering the blue intensity as indicator of the product concentration: it was showed that the polymerization process follows a second order reaction as established by Butler M.F. et al. [17,19,32]. The gelatin/collagen solution after GP addition is initially clear and slightly yellow, then it becomes brownish and finally blue. Blue pigments derives from an oxygen radical polymerization of GP and dehydrogenation of intermediate compounds, following ring-opening reaction due to the attack of GP by a primary amine group $[17,19]$. So the radical reaction could be considered the last of a series of crosslinking reactions [2,34]. The $k$ values remains almost constant for gelatin and gelatin-collagen solutions. In fact $k$ depends on the nature of reagents and not on their concentrations: the blue colour formation is demonstrated to be a solid method for monitoring the polymerisation.

Furthermore, several papers have been focused on the use of the GP as crosslinking agent for gelatin [21-24]: the collagen, added in the present work as chemoattractant, could in principle alter the gelling properties of the gel. At the investigated concentrations, no changes have been highlighted on the reaction rate constant, indicating that, for the scaffold fabrication, similar parameters can be used with or without collagen.

It is known that the gelling properties gelatin-GP gel depend on the temperature: at low temperatures the physical formation of the polymer is dominant while at higher temperatures the reaction of GP is more important. In our case, experiments were performed at $37^{\circ} \mathrm{C}$ with the aim at simulating the biofabrication of gel structures embedding cells, or heat sensitive biomolecules. 
Although evaluated with a different algorithm and without the presence of collagen [33], the gelling time of GP-gelatin-collagen gel is in good agree with other values present literature. Furthermore, the results indicated that the fabrication window using robotic dispensing technologies lasts up to 40 minutes at least, that is more than enough for building also complex structures [27].

The static and dynamic diffusion coefficients were determined in order to use this polymeric system with a 3D printing device [29]. The static coefficient was evaluated in a purposely-designed device, using a polymeric membrane with which the GP does not react, the agarose, while the dynamic diffusion coefficient takes into account the changes into the diffusion matrix induced by the GP reaction.

The static coefficient is higher than the dynamic one, because there were no chemical reactions involved in the diffusion process, while the crosslinking of gelatin membrane obstacles GP diffusion. From a deep analysis of this process we made some remarks. Diffusion is a slow process and in this case after 3 hours the system did not reach equilibrium: it was considered a pseudoequilibrium. Measuring the GP concentration of both chambers at the end of the experiment there was a missing GP weight: part of the GP remained trapped both in agarose (up to $11.8 \%$ ) and in gelatin membrane (up 16.8\%). These phenomena are strictly linked with diffusion coefficient reduction. The slow rate of diffusion can be considered an advantage from $3 \mathrm{D}$ printing point of view: diffusion tends to blur the printed image, decreasing the shape fidelity; by printing a GP solution onto a gelatin-collagen substrate it will be possible to obtain well-defined scaffolds.

\section{Conclusions}

The results presented in this paper indicate that the colour intensity of the blue pigment due to the GP reaction is a good index of polymerization, as the kinetic of pigment change follows the kinetic of the reaction with a good degree of approximation. Rheological analysis shows a quite 
large time window in which the polymeric system formed by GP/gelatin/collagen can be used in robotic dispensing devices for 3D plotting scaffolds. At last, the determination of the diffusion constant could be helpful in the calibration of $3 \mathrm{D}$ inkjet printing systems for the fabrication of scaffolds with complex structures. 


\section{References}

1. Mian M, Beghe F, and Mian E, Int J Tissue React 14 Suppl, 1 (1992).

2. Thomson RC, Wake MC, Yaszemski MJ, and Mikos AG, in Advances in Polymer Science, edited by N. Peppas and R. Langer (Springer Berlin Heidelberg, Berlin, Heidelberg, 1995), pp. 245-274274.

3. Brown RA and Phillips JB, in International Review of Cytology, edited by K. W. Jeon (Academic Press, 2007), pp. $75-150$.

4. Bigi A, Cojazzi G, Panzavolta S, Rubini N, and Roveri K, Biomaterials 22, 763 (2001).

5. Bigi A, Cojazzi G, Panzavolta S, Roveri N, and Rubini K, Biomaterials 23, 4827 (2002).

6. Digenis GA, Gold TB, and Shah VP, J. Pharm. Sci. 83, 915 (1994).

7. Miyata T, Taira T, and Noishiki Y, Collagen Biomaterials 9, 139 (1992).

8. Kuijpers AJ, Engbers GHM, Krijgsveld J, Zaat SAJ, Dankert J, and Feijen J, Journal of Biomaterials Science, Polymer Edition 11, 225 (2000).

9. Panduranga Rao K, Journal of Biomaterials Science, Polymer Edition 7, 623 (2013).

10. Nishi C, Nakajima N, and Ikada Y, J. Biomed. Mater. Res. 29, 829 (1995).

11. Lohre JM, Baclig L, Wickham E, Guida S, Farley J, Thyagarajan K, Tu R, and Quijano RC, Asaio J. 39, 106 (1993).

12. Sung HW, Huang RN, Huang LL, and Tsai CC, Journal of Biomaterials Science, Polymer Edition 10, 63 (1999).

13. Sung HW, Chang Y, Chiu C-T, Chen C-N, and Liang H-C, Biomaterials 20, 1759 (1999). 
14. Sung HW, Chen CN, Huang RN, Hsu JC, and Chang WH, Biomaterials 21, 1353 (2000).

15. Sung HW, Huang RN, Huang LL, Tsai CC, and Chiu CT, J. Biomed. Mater. Res. 42, 560 (1998).

16. Tseng T-H, Chu C-Y, Huang J-M, Shiow S-J, and Wang C-J, Cancer Lett. 97, 61 (n.d.).

17. Mi F-L, Sung H-W, and Shyu S-S, J. Polym. Sci. a Polym. Chem. 38, 2804 (2000).

18. Liang HC, Chang WH, Liang HF, Lee MH, and Sung HW, Journal of Applied Polymer Science 91, 4017 (2004).

19. Touyama R, Takeda Y, Inoue K, Kawamura I, Yatsuzuka M, Ikumoto T, Shingu T, Yokoi T, and Inouye H, Chem. Pharm. Bull. 42, 668 (1994).

20. Touyama R, Inoue K, Takeda C, Yatsuzuka M, Ikumoto T, Moritome N, Shingu T, Yokoi T, and Inouye H, Chem. Pharm. Bull. 42, 1571 (1994).

21 Cheng NC, Estes BT, Young TH, Guilak F Tissue Eng Part A.;19(3-4):484-96 (2013)

22 Focaroli S, Teti G, Salvatore V, Durante S, Belmonte MM, Giardino R, Mazzotti A, Bigi A, Falconi M. Microsc Res Tech. 77(11):928-34. (2014)

23 Saglam A, Perets A, Canver AC, Li HL, Kollins K, Cohen G, Fischer I, Lazarovici P, Lelkes PI. J Mol Neurosci.49(2):334-46. (2013)

24 Urciuolo A, Quarta M, Morbidoni V, Gattazzo F, Molon S, Grumati P, Montemurro F, Tedesco FS, Blaauw B, Cossu G, Vozzi G, Rando TA, Bonaldo P. Nat Commun. 4:1964. (2013)

25 Jelen C, Mattei G, Montemurro F, De Maria C, Mattioli-Belmonte M, Vozzi G. Mater Sci Eng C Mater Biol Appl. 33(1):28-36. (2013) 
26 Vozzi G, Corallo C, Carta S, Fortina M, Gattazzo F, Galletti M, Giordano N. J Biomed Mater Res A. 102(5):1415-21 (2014)

27. Malda J, Visser J, Melchels FP, Jüngst T, Hennink WE, Dhert WJ, Groll J, Hutmacher DW.

Advanced Material. 25;25(36):5011-28 (2013).

28. Tirella, A, C De Maria, G Criscenti, G Vozzi, and A Ahluwalia. Rapid Prototyping Journal. 18 (4): 299-307 (2012).

29. C. De Maria, J. Rincon, A. A. Duarte, G. Vozzi, and T. Boland, Polym. Adv. Technol. 24, 895 (2013).

30. B. Derby, Annu. Rev. Mater. Res. 40, 395 (2010).

31. T. Elsdale and J. Bard, J. Cell Biol. 54, 626 (1972).

32. Butler MF, Ng YF, and Pudney PDA, J. Polym. Sci. a Polym. Chem. 41, 3941 (2003).

33. Nickerson MT, Patel J, Heyd DV, Rousseau D, Paulson AT. International Journal of Biological Macromolecules 2006 39: 298-302

34. Sykes P, A Guidebook to Mechanism in Organic Chemistry, 6/E (Pearson Education India, London, 1986), pp. 238-242. 


\section{Figure Legend}

Figure 1: Diffusion device: general scheme where the septum (S), larger chamber (LC) and diffusion chamber (DC) are indicated (a). The septum is composed of three parts: part A, made in Teflon, the part $\mathrm{B}$, a thin silicone film which supports the membrane, and part $\mathrm{C}$, in Plexiglas, to hold the part B pressed against the part A. All these parts had in the central area a window to allow diffusion. On A and C, a large mesh gauze, to not interfere in the measurement, was glued to confine the membrane in the septum.

Figure 2: Linear regression for 5\% gelatin samples crosslinked with different GP concentrations.

Figure 3: Linear regression for $5 \%$ gelatin $+2 \mathrm{mg} / \mathrm{ml}$ collagen 1:1 samples crosslinked with different GP concentrations.

Figure 4: k value for gelatin and collagen polymeric systems for different GP concentrations.

Figure 5: gelling time as function of GP concentration

Figure 6: viscosity over the time as function of GP concentration

Figure 7: GP concentrations in the DC versus time and fitting curve, in case of $1 \% \mathrm{w} / \mathrm{v}$ agarose membrane; $0.25 \% \mathrm{w} / \mathrm{v}$ initial GP concentration (a); $0.5 \% \mathrm{w} / \mathrm{v}$ initial GP concentration in the LC (b).

Figure 8: GP concentrations in the DC versus time and fitting curve, in case of $5 \%$ w/v gelatin membrane; $0.25 \% \mathrm{w} / \mathrm{v}$ initial GP concentration (a); $0.5 \% \mathrm{w} / \mathrm{v}$ initial GP concentration in the LC (b). 


\section{Appendix - Supporting information}

The diffusion system follows the Fick's laws, and at the steady state, the flux can be considered constant within the membrane. This hypothesis is supported by the small thickness of the membrane. The number of particles $\mathrm{N}$ which passes through an unit area in an unit time, or flux, $\mathrm{J}$ can be expressed, at the steady state as:

$$
J=-D \frac{d C}{d x}=\frac{D}{L}\left(C_{1}-C_{2}\right)
$$

The number of molecules in the system is constant, so:

$$
V_{1} C_{1}+V_{2} C_{2}+V_{m} C_{m}=\text { constant }
$$

where $V_{m}$ was the volume of membrane and $C_{m}$ the GP concentration within the membrane itself. Considering a small variation of the elements of equation 2 it resulted equal to zero:

$$
d\left(V_{1} C_{1}\right)+d\left(V_{2} C_{2}\right)+d\left(V_{m} C_{m}\right)=0
$$

The membrane volume is negligible respect to $V_{1}$ and $V_{2}$, and in addition $V_{1}, V_{2}$ and $V_{m} \operatorname{did}$ not vary during the experiment, thus eq. 4 can be assumed:

$$
V_{1} d C_{1}=-V_{2} d C_{2}
$$

integrating equation 10 :

$$
\int_{C_{1}}^{C_{11}} V_{1} d C_{1}=-\int_{0}^{C_{2}} V_{2} d C_{2}
$$

where $\mathrm{C}_{1}$ was the starting concentration of GP solution and $\mathrm{C}_{11}$ the final concentration in the LC. The concentration in the DC at the beginning of the experiment is zero, while final concentration is $\mathrm{C}_{2}$. Solving equation 5: 


$$
V_{1} C_{11}-V_{1} C_{1}=-V_{2} C_{2}
$$

The equations that describe mass balance of GP in the two chambers are:

$$
\left\{\begin{array}{l}
V_{1} \frac{d}{d t}\left(C_{1}\right)=-J_{1} A \\
V_{2} \frac{d}{d t}\left(C_{2}\right)=-J_{2} A
\end{array}\right.
$$

where $\mathrm{J}_{1}$ and $\mathrm{J}_{2}$ are the fluxes from the LC and from the DC respectively. Applying the approximation of quasi steady state,

$$
J_{2}=-J_{1}=J
$$

Generally this approximation is valid if the characteristic time for the system to reach the equilibrium ( $\left.\tau_{\text {eq }}\right)$ was bigger that the characteristic time for the flux to reach the steady state $\left(\tau_{\mathrm{ss}}\right)$. The $\tau_{\text {eq }}$ can be deduced from equation 7 :

$$
\tau_{e q}=\frac{C_{1} V_{1}}{J_{1} A}=\frac{L(A \cdot h)}{D A}=\frac{L h}{D}
$$

where $h$ is the length of the chamber (obtained by dividing the volume by the diffusion surface A), while the $\tau_{\mathrm{ss}}$, as each diffusion phenomenon, is:

$$
\tau_{s s}=\frac{L^{2}}{D}
$$

By comparing equation 9 and 10,

$$
\tau_{s s} \ll \tau_{e q} \Rightarrow \frac{\mathrm{L}}{h} \ll 1
$$

From equation 7 , 


$$
J \cdot A=\frac{D \cdot A}{L} \cdot\left(C_{1}-C_{2}\right)=-V_{1} \cdot \frac{d C_{1}}{d t}=V_{2} \cdot \frac{d C_{2}}{d t}
$$

the molecules flow from $V_{1}$ per second or those coming in $V_{2}$ per second is thus obtained. From equation 6:

$$
C_{11}=\frac{V_{1} C_{1}-V_{2} C_{2}}{V_{1}}
$$

and replacing it in equation 12 :

$$
\begin{gathered}
\frac{D \cdot A}{L} \cdot \frac{V_{1} C_{1}-V_{2} C_{2}}{V_{1}}-C_{2}=V_{2} \cdot \frac{d C_{2}}{d t} \\
\frac{V_{1} C_{1}-\left(V_{1}+V_{2}\right) \cdot C_{2}}{V_{1} V_{2}} \frac{D A}{L} d t=d C_{2} \\
\frac{D \cdot A}{L} \cdot \frac{1}{V_{1} V_{2}} d t=\frac{1}{V_{1} C_{1}-\left(V_{1}+V_{2}\right) \cdot C_{2}} d C_{2}
\end{gathered}
$$

integrating eq. 22:

$$
\begin{gathered}
\int_{0}^{t} \frac{D \cdot A}{L} \cdot \frac{1}{V_{1} V_{2}} d t=\int_{0}^{C_{2}} \frac{1}{V_{1} C_{1}-\left(V_{1}+V_{2}\right) \cdot C_{2}} \\
\frac{D \cdot A}{L} \cdot \frac{1}{V_{1} V_{2}} t=\frac{1}{-\left(V_{1}+V_{2}\right)}\left[\ln \left(V_{1} C_{1}-\left(V_{1}+V_{2}\right) \cdot C_{2}\right)-\ln \left(V_{1} C_{1}\right)\right] \\
-\frac{D \cdot A}{L} \cdot \frac{V_{1}+V_{2}}{V_{1} V_{2}} t=\ln \left(\frac{V_{1} C_{1}-\left(V_{1}+V_{2}\right) C_{2}}{V_{1} C_{1}}\right) \\
e^{\frac{D \cdot A}{L} \cdot \frac{V_{1}+V_{2}}{V_{1} V_{2}} t}=1-\frac{\left(V_{1}+V_{2}\right) C_{2}}{V_{1} C_{1}}
\end{gathered}
$$

from eq.20, $\mathrm{C}_{2}$ can be deduced: 


$$
C_{2}=\frac{V_{1} C_{1}}{V_{1}+V_{2}} \cdot\left(1-e^{\frac{D \cdot A}{L} \cdot \frac{V_{1}+V_{2}}{V_{1} V_{2}} t}\right)
$$

